\title{
MR Investigation of the Coupling between Myocardial Fiber Architecture
}

\section{and Cardiac Contraction}

\author{
Yin $\mathrm{Wu}^{1,2,3,4}$, and $\mathrm{Ed} \mathrm{X} . \mathrm{Wu}^{1,2}$ \\ ${ }^{1}$ Dept. of Electrical and Electronic Engineering, ${ }^{2}$ Laboratory of Biomedical Imaging and Signal Processing, \\ The University of Hong Kong, Hong Kong \\ ${ }^{3}$ Paul C. Lauterbur Research Center for Biomedical Imaging, Institute of Biomedical and Health Engineering, \\ Shenzhen Institute of Advanced Technology, ${ }^{4}$ Key Laboratory of Biomedical Informatics and Health \\ Engineering, Chinese Academy of Sciences, Shenzhen, China
}

\begin{abstract}
Left ventricular structure has proved to be related with cardiac function; however the relation of myocardial fiber distribution with regional wall motion remains to be elucidated. In this study, both in vivo tagging and ex vivo DTI studies were performed in adult rats. $L V$ circumferential strain, myocardium twist and myocardial fiber architecture were investigated. Results show that myocardial fiber distribution has direct relation with LV myocardium magnitude of circumferential strain and twist angle. Such integrated functional and structural analysis may provide more information for understanding the fundamental cardiac mechanics and assessment of pathological changes.
\end{abstract}

\section{INTRODUCTION}

Precise quantitative description of left ventricle (LV) regional wall motion and deformation is necessary to better understand cardiac mechanics. Numerous studies have been performed to examine transmural myocardium deformation with markers implanted in LV wall (1-3). Recently, MR tagging has been widely used to quantify regional myocardium wall motion (4-7). Such mechanical properties as ventricular torsion, strain, and stress are known to be determined by the myocardial fiber structure of LV (8-10). Thus, a precise knowledge of LV myocardial fiber structure can reveal the cardiac mechanics in both normal and pathologic states.

In recent years, diffusion tensor magnetic resonance imaging (DTI) has emerged as a powerful tool for rapid measurement of cardiac geometry and fiber structure at high spatial resolution. Numerous DTI studies explored LV myocardial fiber orientation, results of which have been validated by histological measurements (11-13). These DTI studies show that when viewed from apex, the orientation of LV fibers changes smoothly from a left-handed helix in the epicardium to a right-handed helix in the endocardium, and transmural helix angle typically range from $-60^{\circ}$ to $+60^{\circ}$.

Although the LV structure has been proposed to be associated with cardiac function (14), the relation of myocardial fiber architecture with regional wall motion remains to be elucidated. In this study, in vivo tagging and ex vivo DTI were performed on adult rats. LV myocardium circumferential strain $\left(\mathrm{E}_{\mathrm{CC}}\right)$, twist angle and myocardial fiber architecture were investigated. The relation of myocardial fiber structure with cardiac wall motion was explored.

\section{Methods}

All imaging experiments were conducted on a 7T Bruker PharmaScan (Bruker BioSpin).

\section{A. In vivo MR tagging}

Adult $\mathrm{SD}$ rats $(\mathrm{N}=6)$ were anesthetized with $1.5 \%$ isoflurance. Two sets of SPAMM sequences with orthogonal stripe tags were employed to yield better contrast and higher SNR than single grid tagging dataset. ECG and respiratory triggered tagging imaging were performed on three short-axis slices at apex, middle ventricle and base, respectively. The parameters were: $\mathrm{TR} / \mathrm{TE}=15 / 2.8 \mathrm{~ms}$, slice thickness $=1.5 \mathrm{~mm}$, slice gap $=1.5 \mathrm{~mm}$, cardiac frames $=10$, flip angle $=30^{\circ}, \mathrm{FOV}=6 \times 6 \mathrm{~cm}^{2}$, matrix size $=256 \times 256$, tagging distance $=1.0 \mathrm{~mm}$ with thickness of $0.2 \mathrm{~mm}$, tagged images were zero filled into a $512 \times 512$ data matrix. Fig. 1a shows a tagging image of a slice at middle ventricle. The tagging mesh was traced and divided into 
non-overlapping triangular tissue elements (Fig.1b). Regional wall motion was tracked by the displacement of the centroid of each triangle (Fig.1c). Twist angle, defined as the rotation of the centroid of the triangles from end diastole (ED) to end systole (ES) (5), was measured. Positive twist represents clockwise rotation viewed from base. Lagrangian strain tensor was computed in each triangle by use of homogeneous strain analysis, and $\mathrm{E}_{\mathrm{CC}}$ was calculated with Lagrangian strain tensor projected onto the circumferential direction as described in (5).

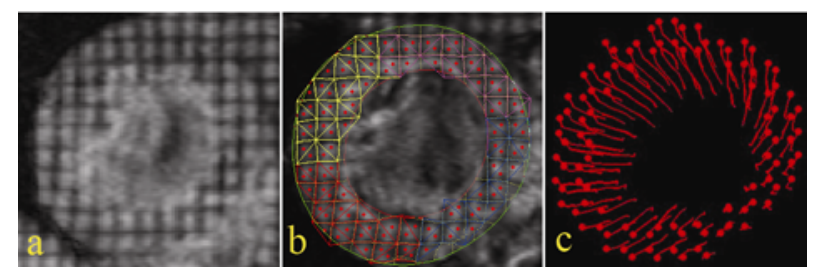

Fig. 1 Tagging mesh (a) was divided into non-overlapping triangular tissue elements with the centroid of each triangle defined (b). Regional wall motion was tracked by the displacement of the centroid of each triangle (c).

\section{B. Ex vivo DTI study}

All animals were sacrificed after tagging study with excised hearts fixed with formalin. DTI was performed at the same three slices using spin echo DTI with parameters: $\mathrm{TR} / \mathrm{TE}=1500 / 29 \mathrm{~ms}$, diffusion $\mathrm{b}=800 \mathrm{~s} / \mathrm{mm}^{2}$, number of gradient directions $=6, \mathrm{FOV}=2.55 \mathrm{~cm}^{2}$, matrix size $=256 \times 256$, and $\mathrm{NEX}=10$. The scan time was $\sim 7 \mathrm{hr}$ per sample. DtiStudio (15) was used to obtain primary eigenvector, from which fiber helix angle was computed with a developed MATLAB program (16-19). Myocardial fibers were then categorized into three types: left-handed helical fiber (LHF), circumferential fiber (CF) and right-handed helical fiber (RHF) based on three different fiber architecture classifications: the break points of LHF and CF were $-20^{\circ},-30^{\circ}$ and $-40^{\circ}$, and the break points of $\mathrm{CF}$ and RHF were $20^{\circ}, 30^{\circ}$ and $40^{\circ}$ for Group \#1, Group \#2 and Group \#3, respectively. In each group, fiber percentages were correlated with the magnitude of $\mathrm{E}_{\mathrm{CC}}$ and twist angle of the three slices. Student's t-test was performed with $\mathrm{p}<0.05$ regarded as significance.

\section{Results}

\section{A. Cardiac regional wall motion}

The LV average magnitude of $\mathrm{E}_{\mathrm{CC}}$ was observed to decreased significantly from apex $(-0.21 \pm 0.01)$, middle ventricle $(-0.18 \pm 0.01)$ to base $(-0.15 \pm 0.01)$ (Fig.2a). Twist angles were found to change substantially from clockwise at apex $\left(14.7 \pm 1.9^{\circ}\right)$ and middle ventricle $\left(4.7 \pm 2.0^{\circ}\right)$ to counterclockwise at base $\left(-4.5 \pm 2.3^{\circ}\right)$ (Fig.2b).

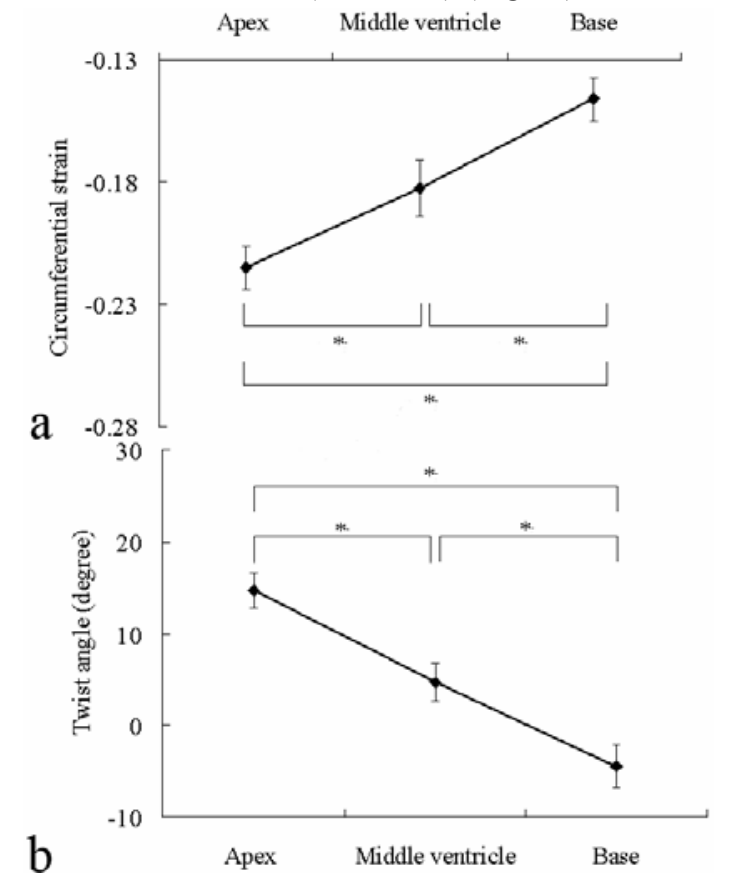

Fig.2 LV circumferential strains (a) and twist angles (b) at apex, middle ventricle and base. ${ }^{*} p<0.05$.

\section{B. Myocardial fiber architecture}

Helix angle maps at apex, middle ventricle and base of a sample was illustrated in Fig.3. Percentages of three types of fibers with different fiber architecture classification were computed and compared in Fig.4. For each fiber architecture classification group, LHF was found to increase significantly from apex to base, while RHF decreased substantially. But no significant change of CF was observed among the three slices.
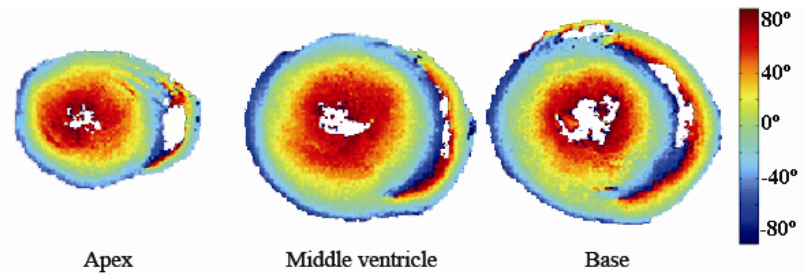

Fig.3 Helix angle maps at apex, middle ventricle and base.

\section{Relation of myocardial fiber structure with cardiac regional wall motion}

Correlations of fiber architecture with LV myocardium wall motion were summarized in Table 1. For each group, 
LHF percentage negatively correlated with twist angle; and RHF percentage positively correlated with twist angle. Therefore, increasing of component difference between RHF and LHF leaded to LV twist more towards clockwise. For CF, no significant correlation was obtained, which may due to its relatively constant values among the three slices. These results directly indicate that RHF and LHF force LV myocardium to twist at opposite direction, and their component difference determines the twist orientation of the slice. Similar results were found for the relations between fiber percentages and average magnitudes of $\mathrm{E}_{\mathrm{CC}}$. The magnitude of $\mathrm{E}_{\mathrm{CC}}$ increased with RHF percentage, but decreased with LHF percentage, suggesting that RHF bundle is another crucial factor in maintaining circumferential shortening besides of CF bundle.
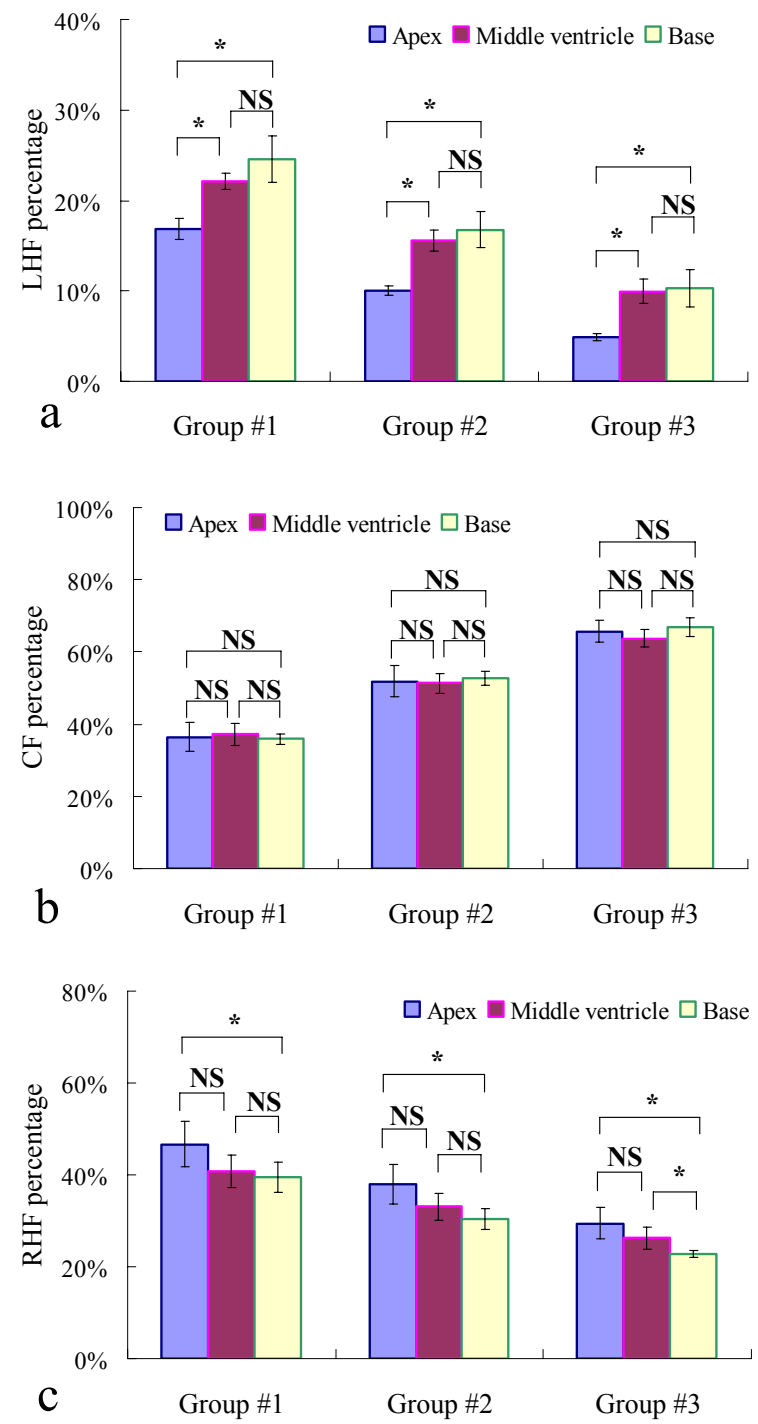

Fig.4 Percentages of LHF (a), CF (b) and RHF (c) at 3 slices with different fiber architecture classifications.
Table 1. Correlations of fiber architecture with LV myocardium wall motion. ${ }^{*} p<0.05$.

\begin{tabular}{clcc}
\hline & & Twist angle & $\left|\mathrm{E}_{\mathrm{CC}}\right|$ \\
\hline \multirow{2}{*}{$\begin{array}{c}\text { Group } \\
\# 1\end{array}$} & LHF $\left(-90^{\circ} \sim-20^{\circ}\right)$ & $-0.86^{*}$ & $-0.88^{*}$ \\
\cline { 2 - 4 } & $\mathrm{CF}\left(-20^{\circ} \sim+20^{\circ}\right)$ & 0.04 & -0.10 \\
\cline { 2 - 4 } & RHF $\left(+20^{\circ} \sim+90^{\circ}\right)$ & $0.60^{*}$ & $0.70^{*}$ \\
\cline { 2 - 4 } & RHF-LHF & $0.75^{*}$ & $0.82^{*}$ \\
\hline Group & LHF $\left(-90^{\circ} \sim-30^{\circ}\right)$ & $-0.87^{*}$ & $-0.87^{*}$ \\
\cline { 2 - 4 }$\# 2$ & CF $\left(-30^{\circ} \sim+30^{\circ}\right)$ & -0.11 & -0.22 \\
\cline { 2 - 4 } & RHF $\left(+30^{\circ} \sim+90^{\circ}\right)$ & $0.70^{*}$ & $0.77^{*}$ \\
\cline { 2 - 4 } & RHF-LHF & $0.83^{*}$ & $0.88^{*}$ \\
\hline Group & LHF $\left(-90^{\circ} \sim-40^{\circ}\right)$ & $-0.84^{*}$ & $-0.83^{*}$ \\
\cline { 2 - 4 }$\# 3$ & CF $\left(-40^{\circ} \sim+40^{\circ}\right)$ & -0.12 & -0.18 \\
\cline { 2 - 4 } & RHF $\left(+40^{\circ} \sim+90^{\circ}\right)$ & $0.76^{*}$ & $0.80^{*}$ \\
\cline { 2 - 4 } & RHF-LHF & $0.88^{*}$ & $0.90^{*}$ \\
\hline
\end{tabular}

\section{Discussion AND CONCluSION}

Twist angle and $\mathrm{E}_{\mathrm{CC}}$ of $\mathrm{LV}$ myocardium were assessed by MR tagging, and ex vivo DTI was applied to investigate myocardial fiber structure at apex, middle ventricle and base. Shortening of LHF and RHF caused myocardium to twist in opposite directions due to their opposite spiraling orientation. CF typically spirals within the short-axis slice, thus its shortening causes no dramatic twist. Because fiber shortening is generally homogeneous through the ventricular wall (9), the RHF vs. LHF composition has important effect on LV myocardium twist. In this study, component difference between RHF and LHF was found to correlate well with tagging measurement of LV myocardium twist.

Fiber shortening of helical fibers can be decomposed into circumferential and radial strains. Therefore, $\mathrm{E}_{\mathrm{CC}}$ of a slice is contributed from CF, LHF and RHF. As CF shows no apparent change among short-axis slice locations, the circumferential strain change was mainly determined by LHF and RHF of the slice. It is reported that circumferential strain at the endocardium in known to exceed all other shortening strains, and a very small strain is observed at the epicardium (9), therefore, the overall circumferential strain mainly results from the fibers at endocardium (e.g. RHF). Percentage of RHF was found in this study to positively correlate with magnitude of average $\mathrm{E}_{\mathrm{CC}}$, suggesting the key role of RHF in maintaining circumferential shortening 
besides of CF bundle. This finding may explain the RHF loss concurrent with cardiac failure in infarct patients reported in (20).

In conclusion, myocardial fiber distribution has direct relation with LV wall motion. Such integrated functional and structural analysis may provide more information for understanding the fundamental cardiac mechanics and assessment of pathological changes.

\section{ACKNOWLEDGMENT}

This work was supported by a research grant from Hong Kong Research Grant Council (GRF HKU7794/07M).

\section{REFERENCES}

1. Arts T, Veenstra PC, Reneman RS. Epicardial deformation and left ventricular wall mechanisms during ejection in the dog. The American journal of physiology 1982;243(3):H379-390.

2. Waldman LK, Fung YC, Covell JW. Transmural myocardial deformation in the canine left ventricle. Normal in vivo three-dimensional finite strains. Circulation research 1985;57(1):152-163.

3. Freeman GL, LeWinter MM, Engler RL, Covell JW. Relationship between myocardial fiber direction and segment shortening in the midwall of the canine left ventricle. Circulation research 1985;56(1):31-39.

4. Young AA, Imai $\mathrm{H}$, Chang $\mathrm{CN}$, Axel L. Two-dimensional left ventricular deformation during systole using magnetic resonance imaging with spatial modulation of magnetization. Circulation 1994;89(2):740-752.

5. Liu W, Chen J, Ji S, Allen JS, Bayly PV, Wickline SA, Yu X. Harmonic phase MR tagging for direct quantification of Lagrangian strain in rat hearts after myocardial infarction. Magn Reson Med 2004;52(6):1282-1290.

6. Liu W, Ashford MW, Chen J, Watkins MP, Williams TA, Wickline SA, $\mathrm{Yu} \mathrm{X}$. MR tagging demonstrates quantitative differences in regional ventricular wall motion in mice, rats, and men. American journal of physiology 2006;291(5):H2515-2521.

7. Wu EX, Towe $\mathrm{CW}$, Tang $\mathrm{H}$. MRI cardiac tagging using a sinc-modulated RF pulse train. Magn Reson Med 2002;48(2):389-393.

8. Arts T, Reneman RS, Veenstra PC. A model of the mechanics of the left ventricle. Annals of biomedical engineering 1979;7(3-4):299-318.

9. Rademakers FE, Rogers WJ, Guier WH, Hutchins GM, Siu CO, Weisfeldt ML, Weiss JL, Shapiro EP. Relation of regional cross-fiber shortening to wall thickening in the intact heart. Three-dimensional strain analysis by NMR tagging. Circulation 1994;89(3):1174-1182.

10. Taber LA, Yang M, Podszus WW. Mechanics of ventricular torsion. Journal of biomechanics 1996;29(6):745-752.

11. Holmes AA, Scollan DF, Winslow RL. Direct histological validation of diffusion tensor MRI in formaldehyde-fixed myocardium. Magn Reson Med 2000;44(1):157-161.

12. Hsu EW, Muzikant AL, Matulevicius SA, Penland RC, Henriquez CS. Magnetic resonance myocardial fiber-orientation mapping with direct histological correlation. The American journal of physiology 1998;274(5 Pt 2):H1627-1634.

13. Scollan DF, Holmes A, Winslow R, Forder J. Histological validation of myocardial microstructure obtained from diffusion tensor magnetic resonance imaging. The American journal of physiology 1998;275(6 Pt 2):H2308-2318.

14. Sengupta PP, Korinek J, Belohlavek M, Narula J, Vannan MA, Jahangir A, Khandheria BK. Left ventricular structure and function: basic science for cardiac imaging. Journal of the American College of Cardiology 2006;48(10):1988-2001.

15. Jiang H, van Zijl PC, Kim J, Pearlson GD, Mori S. DtiStudio: resource program for diffusion tensor computation and fiber bundle tracking. Computer methods and programs in biomedicine 2006;81(2):106-116.

16. Wu EX, Wu Y, Nicholls JM, Wang J, Liao S, Zhu S, Lau CP, Tse HF. MR diffusion tensor imaging study of postinfarct myocardium structural remodeling in a porcine model. Magn Reson Med 2007;58(4):687-695.

17. Wu EX, Wu Y, Tang H, Wang J, Yang J, Ng MC, Yang ES, Chan CW, Zhu S, Lau CP, Tse HF. Study of myocardial fiber pathway using magnetic resonance diffusion tensor imaging. Magnetic resonance imaging 2007;25(7):1048-1057.

18. Wu Y, Chan CW, Nicholls JM, Liao S, Tse HF, Wu EX. MR study of the effect of infarct size and location on left ventricular functional and microstructural alterations in porcine models. J Magn Reson Imaging 2009;29(2):305-312.

19. Wu Y, Wu EX. MR study of postnatal development of myocardial structure and left ventricular function. J Magn Reson Imaging 2009.

20. Wu MT, Tseng WY, Su MY, Liu CP, Chiou KR, Wedeen VJ, Reese TG, Yang CF. Diffusion tensor magnetic resonance imaging mapping the fiber architecture remodeling in human myocardium after infarction: correlation with viability and wall motion. Circulation 2006;114(10):1036-1045. 